\title{
Medical Robotics - Not A Golem Anymore
}

\section{Giora Weisz}

New York Presbyterian Hospital, Columbia University Medical Center, and Cardiovascular Research Foundation, New York, USA

When people hear the word "robot" their first association is science fiction as a source of visionary fascinating technology that is going to be part of our daily life. When the Czech Capek brothers wrote their science fiction stories and coined the term robot, they probably based it on the $16^{\text {th }}$ century Jewish-Czech folklore of the Golem of Prague, who was created by the rabbi of Prague (the Maharal) to protect his community from the hostile anti-Semites. In Hebrew, the world Golem is used as a metaphor for a brainless creature or entity that serves man under controlled conditions. The most significant boost to public recognition of the robotic concept was done by Isaac Asimov, who published a series of short stories about robots and contributed the famous three laws of robotic behavior or function. The robots in his stories, like the Golem, were independent machines that replaced human beings for various activities. In the last 4 decades, robotics has penetrated into every industrialized field, from assembling cars to medicine, mainly as part of automatism and manufacturing, replacing human workers in many tasks.

In medicine, the integration of robotics has started with automated laboratory work, but has been slower to be integrated into clinical medicine [1]. In the last few years clinical robotic systems have penetrated almost every medical field and its market is expected to reach $\$ 1.3$ billion by 2016 [2].

Robots vary in shape, size, function, and objectives. Hockstein et al. suggested a useful classification for different types of robots to describe these heterogeneous devices [1]. They characterized robots as automated arms, mobile devices, mills, or tele-robotic devices. Additionally, they can be active, semiactive, or passive [1]. Active robotic devices mean that they are pre-programmed, and perform all their function independently without real-time human control. Semiactive systems and passive robotic devices translate movements from an operator's or surgeon's hands into powered or unpowered movements. The semi-active robotics are a tool in the physician hands to provide an enhanced procedure (precision, reputability). These systems will follow the physician order with the ability to scale up and down if needed but are not intended to replace the doctor.

Active medical robots have been developed by humans to increase precision in various surgical tasks. Examples include the Neuromate that is designed for stereotactic brain procedures [3], and the Robodoc for improved accuracy in performing hip replacement surgery [4].

Passive and semi-active robots dominate the surgical robotic arena. With these robots, the operator sits in a workstation away from the patient and manipulates the surgical devices inside the body, often watching the surgical field through an endoscope. The most famous and widely used surgical robot is the daVinci (Intuitive Surgical). This robotic system tracks and translates the surgeon hand movement into the surgical devices that are inserted into the specific surgical area. The daVinci is FDA approved in the US and has been used mainly for prostatectomy, but also for other urogenital, heart, and throat surgeries [5]. Other systems, working on similar concepts, are expected to further expand this field and improve clinical outcomes.

The obvious next step for such robotic technologies is to integrate with tele-robotics. Tele-presence has become reality with the improvement of the Internet network and information/communication technologies. With faster and more reliable connections, the transfer of large amount of data is feasible. Remote presence (Intouch Health) took telemedicine one step ahead, allowing physicians to watch and interact and manipulate diagnostic components (like a statoscope) on a remote patient, connecting through the internet [6]. Combining such telecommunicating technologies with surgical robotics will enable performing really remote procedures: inaccessible geographical areas with low population unable to be transferred to a medical center, wounded soldiers in combat, sailors on prolonged ocean sails (ships and submarines), and even in space.

Another type of robot is to support patients with disabilities. This spans from artificial robotic limbs to nurse replacement. An example to the latter is the Pearl (Nursebot). This robot is designed to help elderly people in their daily life, routine activities like walking support, bathroom activities, eating, drinking, and medication reminders [7]. Similarly, a robotic wheelchair has been suggested [8].

Medical robots are a big leap for medical technologies. They have multiple advantages for both patients and the operator physicians. Medical robots transform surgical and interventional procedures from art to standard reproducible accomplishments. The robot gives the surgeon additional hands with increased stability. These machines have movements that are calculated and executed in precision of a sub-millimeter to the level of perfection. Some surgical robots have the ability to reach the surgical field in a way that is not accessible to human hands. The surgical and interventional vascular robots have also significant benefits to the operators. The later usually operate the robot while sitting in an ergonomic work-station, obviously easier, and reduces the fatigue and orthopedic problems that are associated with prolonged standing while operating. In the cath-lab, the Corpath 200 vascular robot (Corindus) has been shown to reduce the operator exposure to $\mathrm{x}$-ray radiation by over $95 \%$ [9].

In order to have a complete independent machine that does not need constant manipulation by a physician and can make some decisions, there is need to integrate high level of artificial intelligence. This will get robots closer to the envisioned creatures that do helpful tasks. Advanced artificial intelligence will need the support of health information technologies that are able to create large databases

*Corresponding author: Giora Weisz, MD, Associate Professor of Clinical Medicine, Columbia University, Director of Clinical Cardiovascular Research, Center for Interventional Vascular Therapy, New-York Presbyterian Hospital - Columbia University Medical Center, 161 Fort Washington Ave., 6th Floor, New York, NY 10032, USA, Tel: 212-305 7060; E-mail: gw2128@columbia.edu

Received August 21, 2012; Accepted August 23, 2012; Published August 28 , 2012

Citation: Weisz G (2012) Medical Robotics - Not A Golem Anymore. J Health Med Inform 3:e104. doi:10.4172/2157-7420.1000e104

Copyright: (c) 2012 Weisz G. This is an open-access article distributed under the terms of the Creative Commons Attribution License, which permits unrestricted use, distribution, and reproduction in any medium, provided the original author and source are credited. 
of patients, disease conditions, clinical scenarios, and operational procedure. This accumulated information will be necessary to set the ground for further machine-generated decision making. Multiple software applications to help clinical decision making have been generated over the years, but none has got to the level that can replace the medical practice that combines science with art.

One of the big challenges of the robotic field is the integration in hospital and other health-care facilities. Physicians are conservative, and skilled operators believe they "can do it better" than machines. It will take time, money, training, and patience to get robotics fully integrated. Part of his integration has to be done by informationtechnology personnel that have solid backgrounds in technologies, computing, and knowledge how to incorporate new concept into conservative environment. Patients are expected to be more receptive. As the word robot represent technological advance, many patients will be happy to be treated by the aid of state of the art technology. As engineers and support stuff will be involved in the early implementation of new technology, it will be critical to ensure that Health Insurance Portability and Accountability Act (HIPPA) is not breached.

Robotic technologies are sophisticated and need many years of engineering and development and long regulatory path to get FDA approval. Nonetheless, Robotics in healthcare is one of the big waves of innovations, leaving behind the notion of science fiction. Robots will keep penetrating all medical fields, and be an integral part of every health organization. Technological training of health information technology personnel is needed for full and smooth integration.

\section{References}

1. Hockstein NG, Gourin CG, Faust RA, Terries DJ (2007) A history of robots: from science fiction to surgical robotics. J Robotic Surg 1: 113-118.

2. Seitz $P$ (2011) Medical robots market could grow to \$1.3 billion in 2016 .

3. http://www.renishaw.com/en/neuromate-the-no-1-image-guidedneurosurgical-robot--10712.

4. http://www.robodoc.com/

5. http://www.davincisurgery.com/

6. http://www.intouchhealth.com/

7. Pollack ME, Brown L, Colbry D, Orosz C, Peintner B, et al. (2002) Pearl: A Mobile Robotic Assistant for the Elderly.

8. Galindo C, Gonzalez J, Fernandez-Madrigal JA (2006) Control architecture for human-robot integration: application to a robotic wheelchair. IEEE Trans Syst Man Cybern B Cybern 36: 1053-1067.

9. Weisz G, Metzger DC, Caputo RP, Delgado JA, Marshall JJ, et al. (2012) Safety and Feasibility of Robotic Percutaneous Coronary Intervention - The Multi-Center Percutaneous Robotically-Enhanced Coronary Intervention Study (PRECISE). 